\title{
Pediatric transplantation
}

\author{
Paul M. Colombani ${ }^{\mathrm{a}}$, Stephen P. Dunn ${ }^{\mathrm{b}}$, William \\ E. Harmon ${ }^{c *}$, John C. Magee ${ }^{d}$, Sue V. \\ McDiarmid $^{e}$ and Thomas L. Spray ${ }^{f}$
}

${ }^{a}$ The Johns Hopkins Hospital, Baltimore, MD

${ }^{\mathrm{b}}$ Alfred I. duPont Hospital for Children, Wilmington, DE

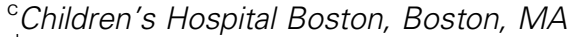

'University of Michigan, Ann Arbor, MI

e University of California Los Angeles, Los Angeles, CA

'The Children's Hospital of Philadelphia, Philadelphia, PA

*Corresponding author: William E. Harmon,

william.harmon@tch.harvard.edu

Key words: Deceased donors, graft survival, living donors, organ donation, patient survival, pediatric transplantation, SRTR, waiting list

\section{Received 17 September 2002, revised and accepted for} publication 26 November 2002

\section{Introduction}

The data presented here are gathered from across all transplant procedures to provide an overview of the state of transplantation among children. The analyses that follow are intended to provide clarity within each area of review regarding patients aged 17 and under, from waiting list registration through 5-year survival of patients and grafts. While it is difficult to draw overall conclusions from a decade's worth of data for six different transplant procedures, a few developments can be identified.

Unless otherwise noted, the statistics in this article come from reference tables in the 2002 OPTN/SRTR Annual Report. Two companion articles in this report, 'Data Sources

Funding: The Scientific Registry of Transplant Recipients (SRTR) is funded by contract \#231-00-0116 from the Health Resources and Services Administration (HRSA). The views expressed herein are those of the authors and not necessarily those of the US Government.

This is a US Government-sponsored work. There are no restrictions on its use.

Note on Sources: The articles in this supplement are based on the reference tables in the 2002 OPTN/SRTR Annual Report, which are not included in this publication. Many relevant data appear in figures and tables directly referred to in the article; other tables from the Annual Report that serve as the basis for this article include the following: Tables 1.1-1.4, 1.8, 2.1-2.7, $2.11,3.2,5.1-5.4,5.7-5.9,6.1,8.1,9.1-9.4,9.8,9.9,10.1-10.4$, $10.8,10.9,11.1-11.4,11.8,11.9,12.1-12.4,12.8,12.9$, and 13.1-13.4. All of these tables are also available online at http:// www.ustransplant.org. and Structure' and 'Analytical Approaches for Transplant Research', explain the methods of the data collection, organization, and analysis that serve as a basis for this article $(1,2)$.

In 2001, the number of pediatric registrants continued to increase in all age groups, except among children younger than 1 year. While the total number of pediatric registrants more than doubled since 1992, parallel growth in the adult population of registrants led to a gradual decline in the relative percentage of pediatric patients among total registrants from a high of 4\% a decade ago, leveling at around 3\% since 1999.

From 1992 to 2001, the greatest increase in pediatric registrants awaiting transplant was observed for the intestine and lung waiting lists, both of which grew approximately fourfold. Currently, the liver waiting list contains the largest number of pediatric registrants, with some 1104 children listed at the end of 2001. Unlike the growth experienced over the decade in other types of pediatric transplantation, the number of children awaiting and receiving kidney transplants has been relatively stable. Given that the incidence of end-stage renal disease (ESRD) has not increased in children in many years, the practice of aggressively identifying and transplanting these patients has kept the number of pediatric kidney transplants steady over the years.

Living donor grafts played a prominent role over the decade. Increased availability of these grafts supported a slight rise in the number of kidney transplants performed in children. Among pediatric recipients of liver transplants, increasing numbers of children aged 11-17 years received living donor grafts.

\section{Pediatric Donor Overview}

In 2001, there were 978 deceased pediatric donors. The absolute number of such donors remained fairly constant over the last decade (range 963-1213). However, the percentage of deceased pediatric donors declined from $23 \%$ in 1993 to $16 \%$ in 2001 because of a significant increase in adult donors above age 35 . It is worth noting that, as with pediatric donors, the number of deceased donors aged 18-34 years also remained relatively constant.

The stability of the total number of pediatric donors over the past decade suggests that initiatives to increase awareness and bolster consent rates remain worth pursuing (3). Additionally, while strides were made over the last decade in accepting more expanded criteria adult donors, it is uncertain to what extent such donors are present in the pediatric population. Finally, it remains to be determined 


\section{Colombani et al.}

how growth in the use of nonheartbeating donors will affect the total number of pediatric donors relative to adult donors.

Figure 1 summarizes the contribution of deceased pediatric donors by organ. In 2001, pediatric donors constituted $16 \%$ of all deceased kidney donors, with the majority of pediatric kidney donors $(n=496)$ aged $11-17$ years. Nearly all pediatric deceased donors were kidney donors, except for those less than 1 year of age, of whom only 48\% donated kidneys in 2001. Overall, pediatric donors were most likely to be kidney donors (92\%), then liver donors (86\%), and heart donors (49\%).

Analysis of pediatric organ donation on the basis of age subgroups revealed that pediatric pancreas and lung donors tended to be older children, while intestine donors were more likely to be aged 5 and younger (Figure 2). Liver and heart donors exhibited roughly even distribution across age groups. Overall, pediatric donors were much more likely to be pancreas, intestine, or heart donors than their adult counterparts; for kidney, liver, and lung donation, contributions between the two groups were relatively well matched (Figure 3 ).

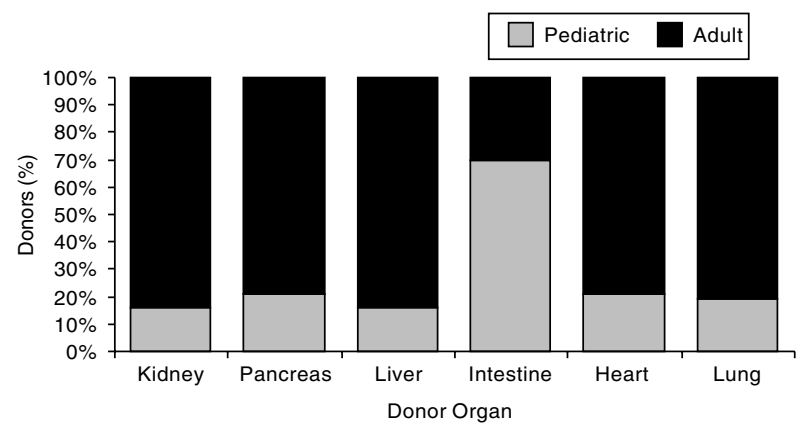

Figure 1: Deceased donors by organ, adult vs. pediatric, 2001. Source: 2002 OPTN/SRTR Annual Report, Tables 2.2-2.7.

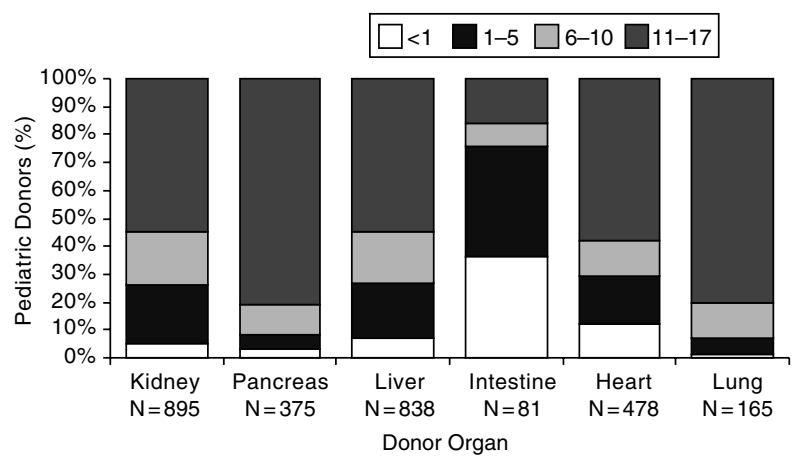

Figure 2: Deceased pediatric donors by organ and age group, 2001. Source: 2002 OPTN/SRTR Annual Report, Tables 2.2-2.7.

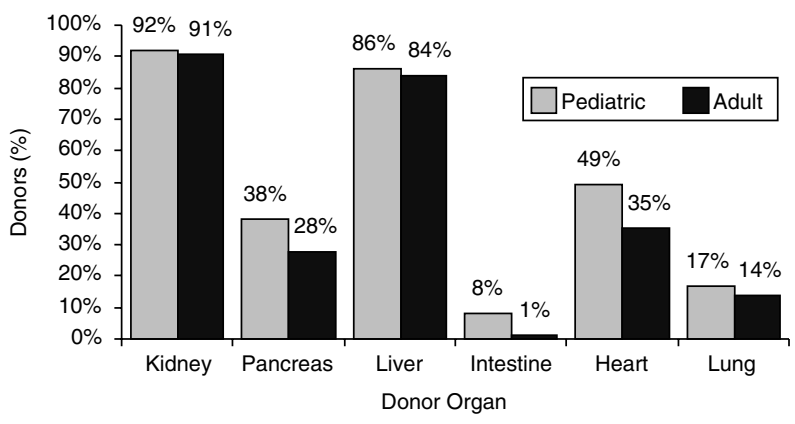

Figure 3: Percent donation of organs of adult and pediatric deceased donors, 2001. Source: 2002 OPTN/SRTR Annual Report, Tables 2.1-2.7.

\section{Kidney Transplantation}

\section{Waiting list}

Over the past four decades, kidney transplantation has become a highly successful treatment for children with ESRD, and the transplant community has come to recognize that indications, endpoints, procedures, complications, pharmacokinetics, and outcomes are different for children than they are for adults. In response to these differences, dedicated pediatric registries, such as the North American Pediatric Renal Transplant Cooperative Study were developed to address the special requirements of children (4). Nonetheless, a comprehensive database such as the SRTR is essential for defining the comparative outcomes of children and adults-and for identifying opportunities to improve the outcomes of both groups.

In 2001, pediatric patients represented $1.4 \%$ of all registrants on the kidney waiting list. Although the number of pediatric patients awaiting renal transplantation continued to increase, the relative percentage of pediatric patients represented on the waiting list declined over the decade from $3 \%$ of all registrants in 1992. This decrease reflects the explosive growth in the number of adult registrants. Of pediatric patients listed for cadaveric renal transplantation in 2001, 69\% were aged 11-17years. Only 1-3 children younger than 1 year were on the waiting list at each year-end snapshot. The relative distribution by age groups remained relatively stable over the decade.

For pediatric waiting list registrations, the median time to transplant was significantly shorter than for adults. For children aged 1-5years and 6-10years, the median time to transplant decreased somewhat in the past 3 years, albeit not drastically. Over the last 10 years, median time to transplant has remained relatively constant or even decreased slightly in all pediatric age groups, unlike the adult age groups, where time to transplant has increased relentlessly.

Annual death rates among pediatric registrants awaiting renal transplantation has remained relatively unchanged 
overall since 1992. Of all registrants on the kidney waiting list, children aged 6-17 had a significantly lower death rate. In contrast, children aged 1-5 demonstrated a significantly higher mortality rate, approaching that observed for older adults (i.e. those aged 35-49 and older).

Without question, renal transplantation is the standard of care for children with ESRD. Unfortunately, a significant shortage of donor organs contributes to waiting list morbidity and mortality. This situation exists despite the virtual absence of donor size limitations that are important to most extrarenal transplants in children. Continued growth of living donor renal transplantation will help continue to relieve pressure on the waiting list.

\section{Waiting list: pancreas and kidney-pancreas}

Currently, pediatric patients are rarely listed for pancreas transplant, and even less frequently for kidney-pancreas. Because there are so few pediatric patients, meaningful analysis of wait time or mortality awaiting transplant is not feasible.

\section{Transplantation and survival}

In the past, young kidney recipients have generally been considered at higher risk, showing diminished graft survival compared with older children and adults $(4,5)$. In many reports, the worst survival rates were seen in the youngest patients, and speculation concerning the causes of these poor results centered around the hypothesis of heightened immune responsiveness in very young children (5). Improvements in surgical technique, donor selection, and immunosuppression practices, as well as the enhanced experience of specialized pediatric transplant teams, have all led to marked improvements in patient and kidney graft survival for infants and young children $(6,7)$. Indeed, recent analyses have identified these patients as now having the best long-term survivals of all age groups $(8,9)$. Young recipients of adult-sized kidneys who have immediate graft function have been reported as having the longest projected graft half-lives, exceeding even those of adult recipients of 2-haplotype matched living donor transplants (10). Adolescent kidney recipients have now replaced infants as having comparatively poor outcomes. The reasons for the increased graft failure in adolescents remain speculative.

The incidence of ESRD in children increased only slightly during the past decade, and this is reflected in the waiting list for kidney transplants. At the end of 1992, 630 children younger than 18 years were on the waiting list for kidney transplants; by the end of 2001 there were 701, an increase of only $11 \%$. In contrast, the number of adults on the list more than doubled, from 21433 to 50443. Importantly, the increases were not seen uniformly throughout the adult age groups. While the number of registrants aged 18-34 increased by $34 \%$ in that time frame, those aged 50-64years increased more than threefold and those over 65 almost sixfold. It would appear the waiting list is indeed aging. Thus, the percentage of those on the list who were children decreased from $3 \%$ to $1 \%$ over the past decade.

The OPTN has always given preference to children awaiting cadaveric renal transplant. Initially, children were preferentially allocated kidneys from deceased donors less than 10 years old. Unfortunately, these donors turned out to be high-risk, and the combination of high-risk donors for high-risk recipients resulted in very poor outcomes for pediatric recipients of cadaveric kidneys $(11,12)$. The allocation system was subsequently changed, first by additional points and then to the present system of placing pediatric patients at the top of the waiting list after waiting times between 6 and 18 months (depending on recipient age). These newer systems unquestionably contributed to improvement in graft outcomes for children and maintained relatively short waiting times. For example, the median waiting time for those aged $1-5$ years at listing was 212 days in 1992 and 205 days in 2001. The waiting times for registrants in other pediatric age groups remained comparably low, while the waiting times of adults increased by a factor of more than two.

The number of children who received kidney transplants increased slightly over the past decade, almost entirely attributable to an increased number of living donor transplants. Of the 592 pediatric kidney transplants performed in 1992, 315 were from living donors, rising to 434 of 723 in 2001. Thus, living donors currently account for $60 \%$ of kidney transplants for children. Children were the recipients of $4 \%$ of all kidney transplants from deceased donors, and $7 \%$ from living donors. Interestingly, in 2001, 898 adults over age 65 received kidneys from deceased donors. Thus, three times as many kidneys were allocated to those over age 65 than to those less than 18, once again emphasizing the aging of the kidney waiting list.

Mortality for children on the waiting list was very low. Only nine children died while awaiting kidney transplantation last year. The lowest mortality was among those aged 6-10 years. Mortality among those younger than 6 was as high as that of patients aged $35-49$ years. Previous studies have demonstrated that mortality in children aged $1-5$ years receiving dialysis is three times higher than for those who receive transplants $(6,13,14)$. The reasons for this unacceptably high mortality are not known, and this area obviously deserves more careful study.

Graft survival rates for pediatric kidney recipients improved substantially during the past decade and now rank among the most successful of all transplants. This outcome may seem surprising given children's poor outcomes previously. Pediatric recipients under age 11 who received living donor kidney transplants had 5-year graft survival rates that were as good or better than most older age groups (92\% for those younger than 1 year, $81 \%$ for those aged 1-5years, and $80 \%$ for those aged 


\section{Colombani et al.}

6-10years) (Figure 4). The results of cadaveric kidney transplants were also slightly better than those seen in adults, with recipients aged $1-5$ years having a 5-year graft survival rate of $68 \%$ and those aged $6-10$ years having the best graft survival rate of all age groups at $72 \%$. These outcomes are consistent with recent reports showing that the longest half-lives of all recipients are in the youngest patients, especially the pediatric recipients of adult-sized grafts who have immediate graft function $(8,10)$. Unfortunately, these excellent results in young children were not seen in adolescent recipients. The 5-year graft survival rate of recipients aged 11-17years who received living donor transplants was $73 \%$, which is roughly equivalent to the results of adult recipients. The adolescent recipients of cadaveric grafts had a 5 -year graft survival rate of $54 \%$. This was worse than rates for all other pediatric and adult age groups except for those aged 65 years and older. The reasons for the poor long-term outcome for the teenage group are unknown, but noncompliance with immunosuppressive medications may play a part. Of course, other causes are also possible, including an unexplained high frequency of graft thrombosis (15) and the high incidence of recurrence of focal segmental glomerulosclerosis (16), which is the most common acquired cause of ESRD in this age group. More recent (1999-2000) recipients of cadaveric grafts in this age group generally had excellent short-term (3-month and 1-year) graft survival rates. Since first-year death rates for 1999-2000 are lower than those for 1995-96 (the cohort of patients for whom 5-year graft and patient survival is reported), it is possible that the longer-term outcomes for this more recent cohort of patients will be better. Until the causes of the diminished graft survival are known, special attention should be paid to this newly identified high-risk teenage group, and using living donors for their kidney transplants should be given strong consideration.

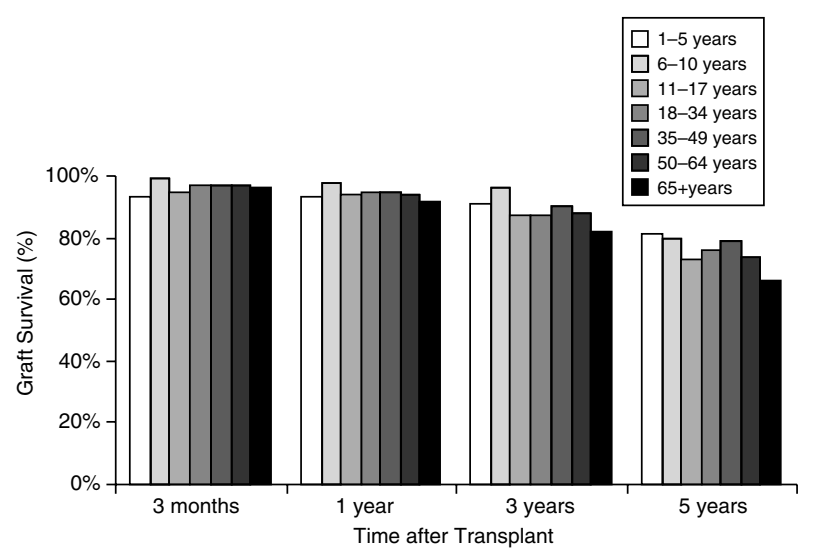

Figure 4: Graft survival among recipients of living donor kidney transplants, by recipient age. Source: 2002 OPTN/SRTR Annual Report, Table 5.8. Cohorts are for transplants performed during 1999-2000 for 3-month and 1-year; 1997-1998 for 3-year; and 1995-1996 for 5-year survival.
As expected, first-year death rates of pediatric kidney transplant recipients were generally lower than those of adults and 5-year patient survival rates were higher. The 5-year patient survival rates for children 1-5, 6-10, and $11-17$ years old were $93 \%, 93 \%$ and $95 \%$, respectively. The youngest recipients, however, may have a slightly higher first-year death rate than older children and young adults. There were 2 deaths in 228 patients $1-5$ years old, resulting in a rate of 18 deaths per 1000 patient years at risk-two to three times higher than older children and young adults. Since the numbers are so small for this age group, however, the significance of the finding is not clear. And, even if the finding is true, it may be difficult to define a cause of the elevated mortality rate.

The use of children as kidney donors has not been well studied. The number of pediatric deceased donors has remained stable over the decade. In 1992, there were 1008 deceased kidney donors under age 18 and in 2001 there were 978. Those numbers represented $22 \%$ and $16 \%$, respectively, of all deceased donors in those years. The current percentage of pediatric deceased donors of kidneys therefore was substantially greater than the percentage of pediatric candidates on the waiting list (1\%) or cadaveric kidney recipients (4\%). Kidneys from donors aged 11-17years had the best short- and long-term graft survival rates, with 5 -year graft survival at $71 \%$. Grafts from younger donors, however, are less successful (11). The 5-year graft survival rate for organs from deceased donors younger than 5 years was $60 \%$, which is equivalent to that for organs from donors aged 50-64 years $(55 \%)$ and superior to those for organs from donors older than $65(42 \%)$. Thus, those very young donors might best be described as expanded criteria donors and the use of their organs for selected recipients should be encouraged. The use of children for living kidney donation remains highly controversial and is generally avoided, except in very limited circumstances, such as identical twins, or in the case of an emancipated minor for his or her own child $(17,18)$. In general, most transplant programs will not accept a living donor younger than 18 years for a kidney transplant.

In summary, over the past decade the picture for graft survival among pediatric renal transplant recipients improved markedly. Certain subgroups of children had the best long-term graft and patient survival rates of all transplant recipients. This success, however, was not uniform among all recipient age groups. Improvements in donor selection, operative techniques, immunosuppressive protocols, and long-term follow-up are all possible methods of improving outcomes. Children are generally transplanted early in the course of ESRD care, since the majority of these patients receive grafts from living donors. Those waiting for cadaveric kidneys experience shorter times to transplant than do adults, since allocation protocols provide them with preference. 


\section{Liver Transplantation}

\section{Waiting list}

Over the past decade, the number of children registered on the waiting list for a liver transplant rose steadily, the most dramatic example being a fivefold increase among those aged 11-17. The number of pediatric registrants rose two- to threefold in the other childhood age groups. Yet even these increases do not match the staggering rise in the number of adults on the liver waiting list. Since 1992, the number of registrants aged 50-64 years awaiting a liver transplant has grown by a factor of 10 . As a result of this huge increase in adults on the waiting list, the relative percentage of children on the list decreased from $16 \%$ in 1992 to $6 \%$ in 2001 . Adults aged $50-64$ years now represent $48 \%$ of all the registrants waiting for liver transplantation. Although patients 5 years or younger receive $6 \%$ of all the liver transplants from deceased donors each year, they represent only $3 \%$ of the waiting list.

It is difficult to compare adult and pediatric patients by liver status because the diseases accounting for transplant are different between the populations, and the status system is not exactly the same for both populations. This especially affects the relative percentages of the $2 \mathrm{~B}$ and 3 categories, which include most of the patients.

For pediatric registrants, the median time to liver transplant was significantly shorter than for adults. The shortest median time to transplant was seen among those aged 6-10 years. Despite these findings, time to transplant generally increased over the last decade for pediatric registrants. It is encouraging to note, however, that since 1999, the median time to transplant decreased among those aged 6-17 years. Unfortunately, time to transplant appears to have increased over the same period for children aged 5 years and younger.

Death while waiting for a transplant has decreased for all pediatric age groups since 1992. These youngest registrants had the highest mortality on the waiting list (572 deaths per 1000 patient years at risk) of any group waiting for a liver transplant, five times the average for all registrants. Death rates for older children ranged between 40 and 162 per 1000 patient years at risk.

The introduction of living donor liver transplantation and the re-emergence of split liver transplantation represent significant advances for pediatric patients $(19,20)$. Nonetheless, given the critical shortage of donor organs, waiting list mortality remains unacceptably high, particularly among children below age 5 . The effect of the recent introduction of the Pediatric End-stage Liver Disease (PELD) score (21) and other changes in organ allocation policy will be monitored closely.

\section{Transplantation and survival}

Over the last decade, an average of 477 cadaveric liver transplants were performed yearly in pediatric patients, with peak activity occurring in 1998 (520 transplants). In 2001, over half of all children transplanted were 5 or younger, and $26 \%$ were 1 or younger.

The proportion of pediatric donor livers that have been transplanted into pediatric recipients has remained fairly constant at around $40 \%$. A recent change in allocation policy for these candidates will direct more pediatric livers toward children and may affect this proportion in the future.

Hepatic allograft survival rates in the pediatric age groups compare favorably with overall allograft survival rates (76-86\% for children vs. $86 \%$ overall) at 3 months. Children younger than 1 year had worse allograft survival in the early postoperative period, but by 5 years were similar to the overall rate $(62 \%$ vs. $64 \%)$. As with kidney transplants, a disturbing trend toward inferior graft survival among patients aged 11-17years at 3 and 5 years posttransplant was notable, when compared to the results for other children and adults. One-year survival was $83 \%$, 3 -year survival was $69 \%$, and 5 -year survival was $60 \%$ in the teenage group. Although these results are from different cohorts, if the 3- and 5-year results persist for the most recent cohort then the rate of decline in graft survival will be steeper for 11-17-year-olds than for most other age groups.

Patient survival for children after liver transplantation compares favorably with adults, except for children under 1 year at 3 months following a liver transplant. At 5 years of follow-up, these youngest children survived at a rate similar to adults (78\% vs. a range of $61-77 \%$ for the adult subgroups). Children aged 6-10 years had the best 5-year survival rate among all patient groups (88\%). Patient survival rates for African Americans were lower than for recipients of other races at all points of follow-up.

The use of living donor grafts has steadily increased. In 1992, 1\% of all liver transplants were from living donors, compared with $10 \%$ in 2001 . Until 1999, pediatric patients constituted most of the recipients of living donor grafts. Since then, however, a major increase in adult-to-adult living donor liver transplantation has occurred, reducing the proportion of living donor transplants accounted for by children. Thus, 97\% of living donor recipients in 1992 were children (younger than 18 years). But by 1999, 39\% of living donor grafts were transplanted into children, and only $21 \%$ of the recipients of living donor grafts were children in 2001. Conversely, the percentage of pediatric recipients who received living donor grafts was only 6\% in 1992. This increased to $14 \%$ in 1997, and its peak to date, $19 \%$, occurred in 2000 as the absolute number of these grafts increased by three times over the course of the decade. 


\section{Colombani et al.}

Until 1998, the majority of all living donor liver grafts went to children under age 1. In 1997, 54\% of all living donor grafts were placed into children in this age range. After 1997, these youngest children still formed the largest group of pediatric living donor recipients. However, in 1999, increasing numbers of older children (11-17 years) began to receive living donor grafts. Of pediatric living donor recipients in 1997, only two of 81 were between ages 11 and 17, but by 2001, the number rose to 18 of 107 pediatric living donor recipients. This paralleled the increasing number of adult living donor recipients, three in 1997 compared with 408 in 2001.

Patient survival for pediatric recipients of cadaveric liver transplants is shown in Figure 5. In comparison, for the cohort of patients transplanted in 1999-2000, 1-year patient survival for pediatric recipients of living donor grafts ranged from $80 \% \quad(n=15)$ for children aged $6-10$ years to $100 \%$ for children aged $11-17$ years $(n=17)$ (Figure 6). For the majority of children receiving living donor grafts - children less than 1 year $(n=85)$ and children aged $1-5$ years $(n=64)$ : 1 -year patient survival rates were $87 \%$ and $83 \%$, respectively. These values remained virtually the same at 3 years, $85 \%$ for both, for the cohort of children receiving transplants between 1997 and 1998.

For the same patient cohorts, 1-year graft survival for living donor grafts transplanted into children was lowest for recipients aged $1-5$ years $(75 \%)$ and highest for recipients younger than age 1 (81\%). However, concordant with results for patient survival, by the third year, graft survival was very similar for all age ranges (77\%). Adequate follow-up for older children is not yet available.

Comparing pediatric with adult recipients of living donor grafts, 1-year patient survival for children receiving such

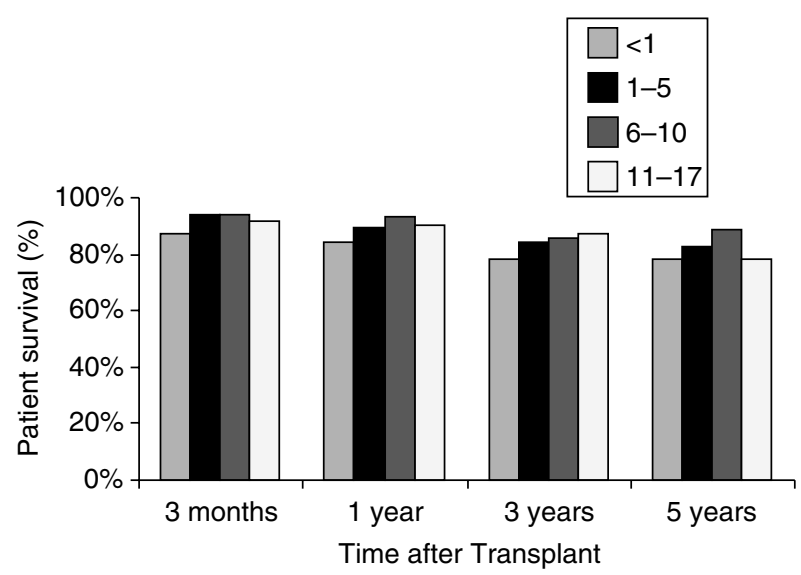

Figure 5: Patient survival among pediatric recipients of deceased donor liver transplants, by recipient age. Source: 2002 OPTN/ SRTR Annual Report, Table 9.9. Cohorts are for transplants performed during 1999-2000 for 3-month and 1-year; 1997-1998 for 3-year; and 1995-1996 for 5-year survival.

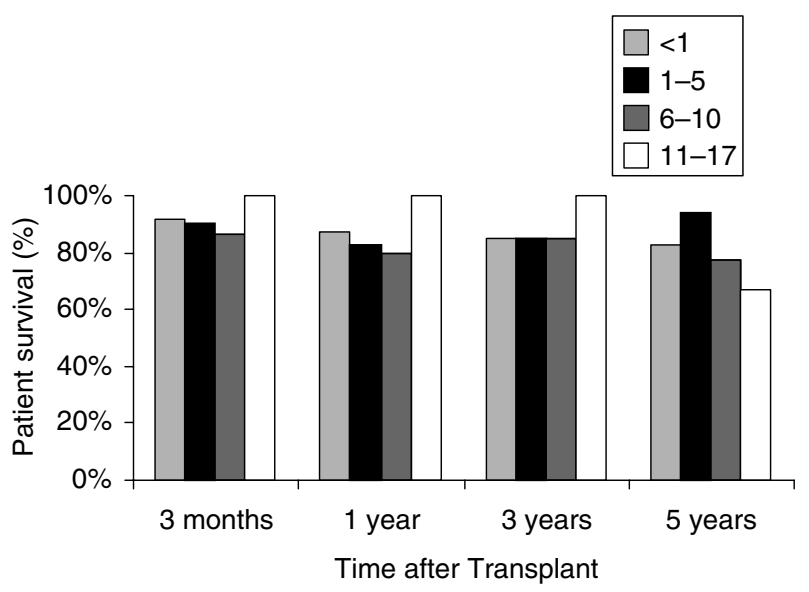

Figure 6: Patient survival among pediatric recipients of living donor liver transplants, by recipient age. Source: 2002 OPTN/ SRTR Annual Report, Table 9.9. Cohorts are for transplants performed during 1999-2000 for 3-month and 1-year; 1997-1998 for 3-year; and 1995-1996 for 5-year survival.

grafts was similar to results for adults receiving them. The lowest 1-year patient survival for adults was in patients older than 65 (76\%); the best 1-year survival was seen in recipients 35-49years old (92\%). Interestingly, adult recipients of living donor grafts tended to have lower 1-year graft survival compared with children. The best survival was reported for recipients aged 35-49years, $79 \%$ for 149 patients; the lowest survival was reported for recipients older than 65, 69\% for 29 patients. Insufficient numbers preclude calculations of three-year graft survival values for adult living donor recipients.

\section{Intestine Transplantation}

\section{Waiting list}

In 2001, 74\% of registrants on the intestine waiting list were 17 years old or younger, and half of these 134 registrants were between ages 1 and 5. Since 1992, the number of pediatric registrants increased approximately fourfold.

Because of relatively small numbers, the median time to transplant is difficult to analyze meaningfully. Despite this limitation, children younger than 1 at time of registration had a strikingly higher median time to transplant compared to other pediatric and adult patients.

Among registrants awaiting intestine transplant, annual death rates were highest in children under 1 year of age, followed by those 1-5year old. Many patients awaiting intestine transplantation are also awaiting a liver transplant. The current MELD/PELD allocation system may not serve this patient population as well as it does those 
with more common etiologies of liver disease, further contributing to waiting list mortality.

As intestine transplantation continues to mature $(22,23)$, it is reasonable to expect continued waiting list growth. The quality of donor organs, along with size considerations and constraints on cold ischemia times, remain limiting factors.

\section{Transplantation and survival}

In 2001, 61 intestinal transplants were performed in pediatric patients. This number is very small compared with numbers for other organ transplants, but it represents a dramatic increase over the 10 intestinal transplants performed in 1992. In 2001, over half of all intestinal transplants performed were in children, and $56 \%$ of these occurred in children aged $1-5$ years.

Patient and graft survival for intestinal transplantation were $62 \%$ and $34 \%$, respectively, at 3 years posttransplant in the group demonstrating the most transplant activity (ages 1-5). Patient survival for pediatric recipients of intestinal transplants by age group is shown in Figure 7 .

\section{Heart Transplantation}

\section{Waiting list}

The number of pediatric registrants listed for heart transplantation has remained relatively stable since the mid-1990s. Little change was seen in the age distribution of new heart waiting list registrations, with the preponderance of pediatric heart transplant registrants aged less than 1 year. This reflects the needs of children with complex congenital heart disease unsuitable for surgical repair or in need of transplantation after failed surgical intervention. The number of new registrations decreased in children aged 6-10 years, reflecting a relatively lower incidence of

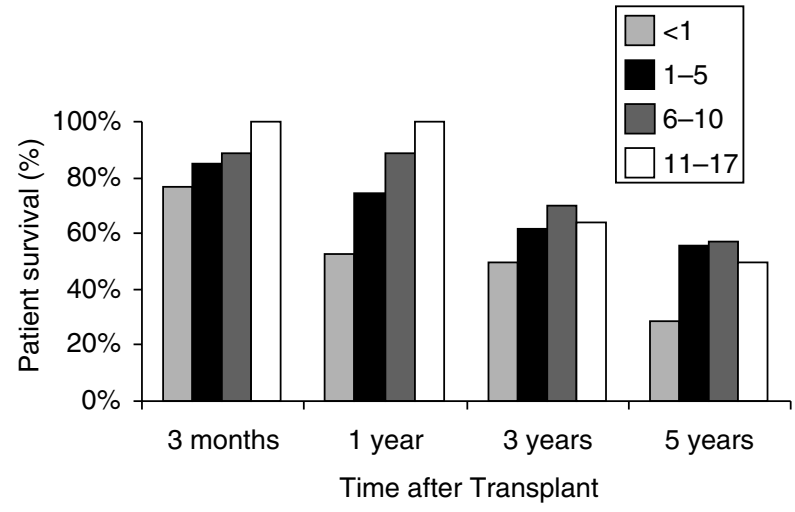

Figure 7: Patient survival among pediatric recipients of deceased donor intestine transplants, by recipient age. Source: 2002 OPTN/ SRTR Annual Report, Table 10.9. Cohorts are for transplants performed during 1999-2000 for 3-month and 1-year; 1997-1998 for 3-year; and 1995-1996 for 5-year survival. cardiomyopathies in this age group. It gradually increased again in those aged 11-17 years, reflecting the effects of end-stage cardiomyopathies and congenital heart disease as an increasing indication for transplantation in this age group. In 2001, cardiomyopathy accounted for $54 \%$ of all registrants, while congenital heart disease accounted for $40 \%$. Coronary artery disease is vanishingly rare among pediatric patients, in contrast to adults.

The median time to heart transplant was substantially lower among pediatric than adult registrants. Since 1999, the median time to transplant among registrants aged 6-10 decreased. Wide variability in waiting times for patients younger than 1 year makes analysis in this subset of patients problematic.

The incidence of death while awaiting heart transplantation was extremely high in registrants under 1 year (1373 waiting list deaths per 1000 patient years at risk). These infants often have complex congenital heart disease, are relatively unstable, and are unable to be supported adequately for longer waiting times. The rate of mortality among this youngest group was almost 10 times the overall waiting list mortality for cardiac transplantation across all age ranges. In children aged 1-5years, waiting list mortality was 377 per 1000 patient years at risk, more than twice the overall mortality for all registrants, and in those aged $6-10$ years the rate of death while waiting was similar to the overall waiting list mortality.

The waiting list death rate was high in registrants aged 11-17 years (279 per 1000 patient years), twice the waiting list death rate for older registrants. The increased rate of death while waiting may reflect the severity of congenital heart disease and cardiomyopathy occurring in children and possibly the need for more accurate size matching, which may result in slightly fewer potential donors for these recipients. The median time to transplant is actually slightly lower for pediatric registrants than for adults. However, the increased waiting list mortality may reflect the instability of the patients and the relatively fewer potential donors for these smaller recipients.

Continued growth in the number of pediatric patients awaiting heart transplantation is likely. The shortage of smaller organs, along with a lack of alternative mechanical support devices for children, contributes to mortality in pediatric patients awaiting transplantation.

\section{Transplantation and survival}

Pediatric cardiac transplantation has been stable in volume since the early 1990 s. Approximately $12 \%$ of all cardiac transplants were performed in patients younger than age 18 , a proportion that was constant over the last 5 years.

There has been a trend over the last decade for more pediatric donor hearts to be transplanted into pediatric recipients (rising from $37 \%$ in 1992 to $49 \%$ in 2001). The 


\section{Colombani et al.}

most recent part of this increase may have resulted in part from an allocation policy change (Policy 3.7.5), which states that within each heart status category, pediatric donor hearts are allocated preferentially to pediatric recipients.

The number of pediatric heart transplant recipients under 1 year of age has slightly decreased over the past 4 years. In 2001, 64 patients received a transplant at or below 1 year, down from 95 in 1997 and a high of 107 in 1993. The number of recipients aged $1-5$ years increased slightly to a decade high of 82 in 2001, possibly reflecting increased activity in transplantation for congenital heart disease that has not improved with surgical reconstruction or palliation. The number of recipients aged 6-10years decreased from 47 in 2000-28 in 2001, the lowest number since 1992. The number of recipients aged 11-17 years has remained relatively constant during the past 5 years at approximately 80-100 patients per year.

Graft survival rates for pediatric heart transplant recipients show a higher early mortality for patients under 1 year; 3-month survival for this group was $86 \%$ compared with survival rates of $88 \%$ or better in all other age groups. This lower survival likely represents the complexity of transplantation in the first year of life in children with complex congenital heart disease. Surgical reconstruction can fail in these youngest patients, and these infants have often waited a significant time for donor organ availability with unprotected pulmonary blood flow and the consequent development of elevated pulmonary vascular resistance. In spite of the decreased 3-month survival in these youngest recipients, $72 \%$ patient survival at 5 years (in a separate cohort) was actually somewhat higher than the other pediatric age groups. Thus, despite higher early postoperative mortality, late mortality appears to be lower, consistent with the possible immune advantage noted in young infants who undergo cardiac transplantation. Therefore, the long-term improved survival may reflect better graft acceptance with effective immunosuppression in this subgroup of patients. After the first year of age, there appears to be no significant difference in outcome at 5 years compared to other age groups.

Early graft survival continued to be slightly lower in recipients aged $1-5$ years (88\%) compared with older children, possibly suggesting again more difficult cardiac anatomy with failed reconstruction for complex congenital heart disease or severe cardiomyopathy at this early age. Data on graft survival also show that survival after a second transplant is worse than initial transplant survival. Although 3-month survival does not appear significantly different at $84 \%$ vs. $89 \%$, at 5 years the retransplant population has a $49 \%$ survival compared to $69 \%$ in the first transplant group.

Younger donor age (under 18years) is associated with graft survival rates ranging from $85 \%$ to $92 \%$ at 3 months and with rates ranging from $63 \%$ to $74 \%$ at 5 years. These survival rates are slightly better than survival rates with older donor ages and represent the relative advantage of young donor grafts with less risk of coronary disease or other cardiac abnormalities. In keeping with these observations, current OPTN heart allocation policies preferentially direct pediatric organs to pediatric recipients.

Patient survival correlates with graft survival, with slightly lower patient survival in the very youngest recipients at 3 months (Figure 8). At 5 years, survival in this group was higher than several of the older age groups.

Data for the 2001 pediatric heart transplantation population continue to suggest stability in both donor availability and the number of new patients listed yearly. A slight increase in patient registrations occurred among those aged 1-5 years. This suggests that cardiac transplantation is used more frequently as a salvage therapy for patients in whom staged reconstruction for a single ventricle was unsuccessful or who have other complex cardiac malformations. Recent interest has developed in the use of non-ABO matched donors for infant and pediatric cardiac transplantation (24). The effect of the use of ABO mismatched donors on overall graft availability and survival rates is yet to be determined. It is apparent, however, that when the number of new registrants aged under 1 year (178 in 2001) is compared with the number of transplants performed (64 in 2001), organ availability is a significant problem. Data are not available to ascertain the total number of potential infant donor hearts not utilized, since some potential infant donors may not have had any organs procured for transplant in the absence of suitable recipients. However, among 84 donors less than 1 year of age from whom at least one organ was procured, only 59 hearts were procured and transplanted. If substantial numbers of cases involved unavailable suitable

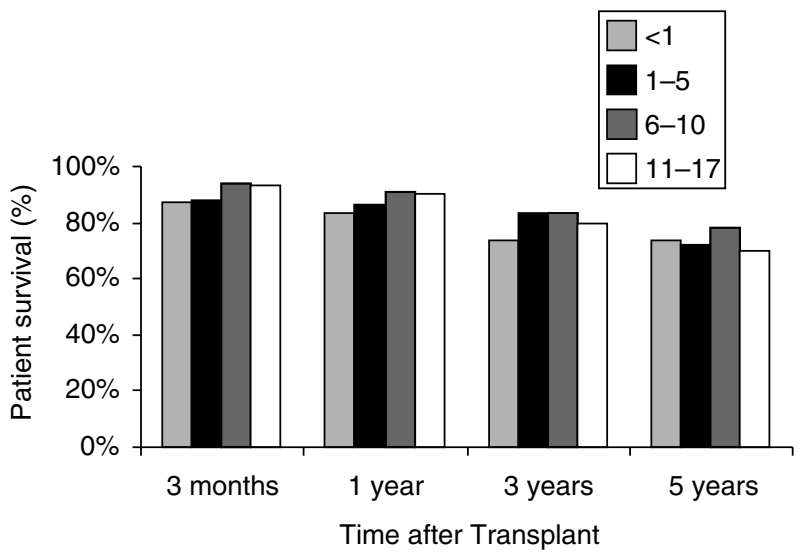

Figure 8: Patient survival among pediatric recipients of deceased donor heart transplants, by recipient age. Source: 2002 OPTN/ SRTR Annual Report, Table 11.9. Cohorts are for transplants performed during 1999-2000 for 3-month and 1-year; 1997-1998 for 3-year; and 1995-1996 for 5-year survival. 
recipients, then $A B O$ mismatching could improve overall transplantation rates and potentially affect survival rates among those awaiting transplant. Any post-transplant effect on patient and graft survival rates at 3 months and 5 years, however, would remain to be determined.

\section{Lung Transplantation}

\section{Waiting list}

Pediatric lung transplantation continues to be an infrequent procedure. New pediatric waiting list registrations for lung transplant peaked in 1998 at 152 and decreased to 109 and 115 in 2000 and 2001, respectively. In children under age 1, only nine new registrations were noted in 2001 , with a similar number in those aged $1-5$ years. The number of registrants aged $6-10$ years also decreased (18 in 2001 vs. 22 in 2000 and 30 in 1999). This pattern also occurred among those registrants aged 11-17years, an age group in which cystic fibrosis is a common indication for transplantation. New registrations peaked in 1998 in this group. The causes for decreased registrations for lung transplants in the pediatric population is unclear. However, improved management of cystic fibrosis, which allows patients to achieve older ages before listing for transplant, may account for some of the decrease. In addition, the contribution of living donor lobar transplantation in the pediatric population with cystic fibrosis must be also considered. The number of lung lobe donors peaked in 1999 at 56, then decreased in 2000 to 30 and in 2001 to 37 . The majority of donors were $35-49$ years old (25).

Analyses of median waiting times to lung transplant are inconclusive because of the insufficient number of patients followed and the small number of waiting list registrations in younger children. No data on waiting time is noted in patients under age 11. Among those aged 11-17 years, the largest group of pediatric lung transplant registrants, median waiting times are not yet known for 2001 registrants. However, the 10th percentile of time to transplant was 200 days, similar to both 2000 and 1999. These waiting times appear to be slightly longer than the 10th percentile of time to transplant in patients aged 18-34 years and in the older age ranges, suggesting that adolescent recipients appeared to be waiting slightly longer than their adult counterparts for donor organs.

The overall death rates while on the waiting list for the entire lung transplant group was 134 per 1000 patient years at risk in 2001, and represents a continual decline over the decade. Patients aged 1-5years showed an almost threefold higher risk of death while waiting in 2001 (362 per 1000 patient years at risk) (Figure 9). In children aged 6-10years, risk of death while waiting decreased from 231 in 1999 to 89 in 2001. The risk for those aged 11-17 years (123) was slightly lower than the overall group of all lung transplant registrants, but showed a similar declining pattern over the 10 years studied. Thus, the very youngest age groups appear to have an increased

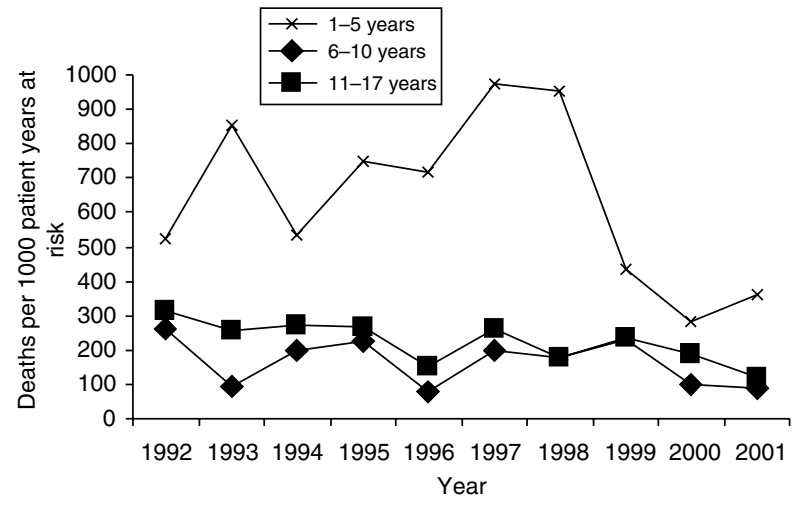

Figure 9: Pediatric lung waitlist death rates per 1000 patient years at risk by recipient age, 1992-2001. Source: 2002 OPTN/ SRTR Annual Report, Table 12.3.

risk of death while waiting, consistent with the limited number of donors suitable for this population (due to size considerations) and the severity of the illnesses for which lung transplantation is considered. The primary indications for lung transplants in young children include congenital malformations of the lung, pulmonary hypertension, surfactant deficiencies, and other severe forms of pulmonary fibrosis. Cystic fibrosis is a very infrequent indication for transplant in this age range. The severity of the lung diseases therefore may increase the risk of death while waiting for organs from an increasingly rare donor pool.

\section{Transplantation and survival}

The number of pediatric lung transplants decreased slightly to 46 in 2001. Out of the 1034 deceased donor lung transplants performed in 2001, 28 were for pediatric patients, representing only $3 \%$ of the total lung transplant population.

Compared to adult recipients, lung graft survival is slightly higher at 3 months for recipients aged $6-10$ years $(90 \%)$ and $11-17$ years $(92 \%)$. Because of the small numbers of patients receiving these grafts, however, the differences in survival rates are not necessarily statistically significant. Five-year graft survival rates in the pediatric population are similar to those for the older patients, varying from $27 \%$ to $50 \%$. The overall graft survival rate was $41 \%$ in the entire lung transplant population.

Patient survival after lung transplantation in pediatric patients appears to be similar to or slightly higher than in the adult population at 5 years (Figure 10). The 5-year survival rate for patients aged less than 1 year at transplant was $33 \%$; in those aged $6-10$ years the survival rate was $64 \%$. For recipients aged $11-17$ years, $45 \%$ of patients were alive at 5 years. Both these measurements have large standard errors allowing for the possibility that overall survival rates are not different from the adult population of lung transplant recipients. Transplantation using a donor 


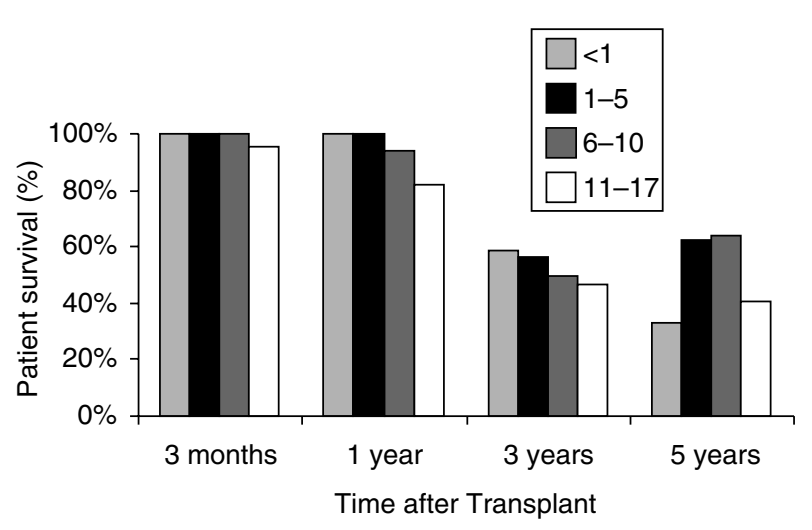

Figure 10: Patient survival among pediatric recipients of deceased donor lung transplants, by recipient age. Source: 2002 OPTN/SRTR Annual Report, Table 12.9. Cohorts are for transplants performed during 1999-2000 for 3-month and 1-year; 1997-1998 for 3-year; and 1995-1996 for 5-year survival.

age less than 1 year appears to be associated with a higher 5-year patient survival rate (54\%) compared to $42 \%$ at 5 years in the overall group. This suggests that the infant recipient of infant lungs may have an advantage, as has been seen for cardiac transplantation in similar age groups.

Lung transplantation in the pediatric population continued to be an unusual procedure with graft and patient survival rates similar to those seen in the adult populations. Very young recipients may possess some shorter-term immune advantage; however, the age cutoff at which this and any improvement in longer-term outcome is noted is still unclear, given the small number of patients transplanted.

Overall, patient survival rates of 5 years are significantly worse than for cardiac transplantation, again reflecting the complexity of lung transplant immunology and the late development of bronchiolitis obliterans in the majority of recipients.

\section{Heart-Lung Transplantation}

\section{Waiting list}

Pediatric registrants made up $17 \%$ of all heart-lung waiting list patients in 2001. In contrast to the heart or lung (alone) waiting lists, the absolute number of pediatric registrants awaiting heart-lung transplantation remained relatively constant and small over the past decade. In 2001, 25 new patients were registered across the entire pediatric age range for this unusual procedure: age less than 1 year $(n=7)$; ages $1-5$ years $(n=4)$; ages $6-10$ years $(n=4)$; and ages $11-17$ years $(n=10)$. These numbers are somewhat decreased from the early 1990s. Deaths and annual death rates per 1000 patient years at risk on the waiting list for heart-lung transplants were significantly increased in pediatric recipients compared with adults. In 2001, the overall waiting list death rate among all heartlung transplant candidates was 191 per 1000 patient years at risk. For children aged 1-5 years, the risk was higher at 232. For those aged 11-17 years, risk was much higher (468) compared with adults aged 18-34 years (159) and with those aged $35-49$ years (143). The reason for the waiting list death rate among those aged 11-17 is not obvious. However, it is possible that these children are in competition for organs with many older patients. The allocation policy for heart-lung recipients may disadvantage children, who are not suitable for assist devices for cardiac support, resulting in a negative impact on overall waiting times and the risk of death while waiting. Unfortunately, there are insufficient data in any of the years to estimate the median waiting time for pediatric heart-lung transplant recipients and therefore this question cannot be directly addressed.

\section{Transplantation and survival}

Of the 27 heart-lung transplants performed in 2001, only six were in children, again showing that heart-lung transplantation is an extremely uncommon procedure in pediatric patients and that the number of potential organs is severely limited. Since 25 pediatric patients were added to the waiting list in 2001 for heart-lung transplant and only six received organs, it is likely that the limited availability of heart-lung blocks in the pediatric sizes accounted for increased waiting list mortality in these age ranges.

Because of the infrequent number of transplants performed in pediatric patients, there are no data available regarding the annual death rates per 1000 patient years at risk for recipients of heart-lung transplants in 2001.

Likewise, few meaningful data are available on graft survival at 3 months, 1 year, 3 years, and 5 years in pediatric heart-lung transplant recipients because of the small number of transplants done. One exception is among recipients aged 11-17years. For these children, the 5-year survival rate of $46 \%$ is similar to the survival rate of the overall population. This suggests indirectly that there is no graft survival disadvantage for pediatric subgroups. As would be expected, patient survival largely mirrors graft survival. Again, the only meaningful data available are for recipients 11-17 year old, who have a 5-year survival rate of $54 \%$. This is slightly better than the overall survival for the entire cohort (49\%).

Heart-lung transplantation continues to be an extremely rare procedure in the pediatric population. The limited indications for heart-lung transplantation, the use of lung transplantation for pulmonary diseases in preference to heart-lung transplant in most centers, and the significant limitation of donor organ availability have limited the use of this procedure. Overall survival rates, however, after heartlung transplantation appear to be similar to those seen in lung transplantation in pediatric patients, although the 
very limited number of transplants make statistically valid comparisons difficult.

\section{Conclusion}

The number of pediatric registrants on the waiting list continued to increase, with the greatest stability seen in the number of such patients awaiting heart and kidney transplants. The greatest percentage increase was observed in the number of pediatric registrants on the intestine and lung waiting lists; the liver waiting list contained the largest absolute number of pediatric registrants. And while the number of pediatric donors remained steady over the last 10 years, the proportion of total donations represented by these children declined to $16 \%$ in 2001 , underscoring the importance of increased awareness about the need for pediatric organ donation. Infants and young children undergoing living and deceased donor kidney transplantation now have the best long-term survival of all age groups. Increasing numbers of children aged 11-17 years received living donor liver grafts, with excellent patient and graft survival rates-though some less encouraging trends also emerged during the study period. Several advances in clinical practices and allocation policies observed during the past decade offer hope for further improvement of outcomes.

\section{References}

1. Dickinson DM, Ellison MD, Webb RL. Data sources and structure. Am J Transplant 2003; 3(Suppl. 4): 13-28.

2. Wolfe RA, Webb RL, Dickinson DM et al. Analytical approaches for transplant research. Am J Transplant 2003; 3(Suppl. 4): 103-113.

3. US General Accounting Office. Organ Transplants: Allocation Policies Include Special Protections for Children. Publication GAO01-498. Washington, DC: US General Accounting Office, 2001.

4. McEnery PT, Stablein DM, Arbus G, Tejani A. Renal transplantation in children. A report of the North American Pediatric Renal Transplant Cooperative Study. N Engl J Med, 1992; 326: 1727-1732.

5. Ettenger RB, Blifeld $\mathrm{C}$, Prince $\mathrm{H}$ et al. The pediatric nephrologist's dilemma: growth after renal transplantation and its interaction with age as a possible immunologic variable. J Pediatr 1987; 111: 1022-1025.

6. Seikaly M, Ho PL, Emmett L, Tejani A. The 12th Annual Report of the North American Pediatric Renal Transplant Cooperative Study: renal transplantation from 1987 through 1998. Pediatr Transplant 2001; 5: 215-231.

7. Tejani A, Sullivan EK, Fine RN, Harmon W, Alexander S. Steady improvement in renal allograft survival among North American children: a five year appraisal by the North American Pediatric Renal Transplant Cooperative Study. Kidney Int 1995; 48: 551-553.
8. Cecka JM, Gjertson DW, Terasaki PI. Pediatric renal transplantation. A review of the UNOS data. Pediatr Transplant 1997; 1: 55-64.

9. Briscoe DM, Kim MS, Lillehei C, Eraklis AJ, Levey RH, Harmon WE. Outcome of renal transplantation in children less than two years of age. Kidney Int 1992; 42: 657-662.

10. Sarwal MM, Cecka JM, Millan MT, Salvatierra O Jr. Adult-size kidneys without acute tubular necrosis provide exceedingly superior long-term graft outcomes for infants and small children: a single center and UNOS analysis. United Network for Organ Sharing. Transplantation 2000; 70: 1728-1736.

11. Harmon WE, Alexander SR, Tejani A, Stablein D. The effect of donor age on graft survival in pediatric cadaver renal transplant recipients - a report of the North American Pediatric Renal Transplant Cooperative Study. Transplantation 1992; 54: 232-237.

12. Harmon WE, Stablein D, Alexander SR, Tejani A. Graft thrombosis in pediatric renal transplant recipients. A report of the North American Pediatric Renal Transplant Cooperative Study. Transplantation 1991; 51: 406-412.

13. Wood EG, Hand M, Briscoe DM et al. Risk factors for mortality in infants and young children on dialysis. Am J Kidney Dis 2001; 37: 573-579.

14. Warady BA, Sullivan EK, Alexander SR. Lessons from the peritoneal dialysis patient database. a report of the North American Pediatric Renal Transplant Cooperative Study. Kidney Int 1996; 53 (Suppl): S68-S71.

15. Singh A, Stablein D, Tejani A. Risk factors for vascular thrombosis in pediatric renal transplantation. a special report of the North American Pediatric Renal Transplant Cooperative Study. Transplantation 1997; 63: 1263-1267.

16. Baum MA, Stablein DM, Panzarino VM, Tejani A, Harmon WE, Alexander SR. Loss of living donor renal allograft survival advantage in children with focal segmental glomerulosclerosis. Kidney Int 2001; 59: 328-333.

17. Delmonico FL, Harmon WE. The use of a minor as a live kidney donor. Am J Transplant 2002; 2: 333-336.

18. Abecassis $\mathrm{M}$, Adams $\mathrm{M}$, Adams $\mathrm{P}$ et al. Consensus statement on the live organ donor. JAMA 2000; 284: 2919-2926.

19. Broering DC, Mueller L, Ganschow R et al. Is there still a need for living-related liver transplantation in children? Ann Surg 2001; 234: 713-721, discussion 721-722.

20. Farmer DG, Yersiz H, Ghobrial RM et al. Early graft function after pediatric liver transplantation. comparison between in situ split liver grafts and living-related liver grafts. Transplantation 2001; 72: 1795- 1802.

21. Wiesner RH, McDiarmid SV, Kamath PS et al. MELD and PELD. application of survival models to liver allocation. Liver Transplantation 2001; 7: 567-580.

22. Abu-Elmagd $\mathrm{K}$, Reyes $\mathrm{J}$, Bond $\mathrm{G}$ et al. Clinical intestinal transplantation. A decade of experience at a single center. Ann Surg 2001; 234: 404-416, discussion 416-417.

23. Kaufman SS, Atkinson JB, Bianchi A et al. Indications for pediatric intestinal transplantation. A position paper of the American Society of Transplantation. Pediatr Transplant 2001; 5: 80-87.

24. West LI, Pollock-Barziv SM, Dipchand Al et al. ABO-incompatible heart transplantation in infants. N Engl J Med 2001; 344: 793-800.

25. Liou TG, Adler FR, Cahill BC et al. Survival effect of lung transplantation among patients with cystic fibrosis. JAMA 2001; 286: 2683-2689. 